\title{
LES RESSOURCES ET LES BLOCAGES DE LA FÉMINISATION DES NOMS EN FRANÇAIS
}

Le choix de féminiser les noms de métiers, titres, grades et fonctions ${ }^{1}$ est un mouvement socialement justifié et qui n'est pas sans avoir de conséquences linguistiques. Seuls, de nos jours, les conservateurs semblent s'y opposer en avançant la plupart du temps des arguments de faible pertinence et parfois saugrenus. Il est vrai aussi que bon nombre de femmes s'accrochent au masculin pour y gagner en prestige. Grâce à la contribution des auteurs du Guide d'aide à la féminisation on ne saurait plus hésiter à favoriser la levée de l'occultation du «sexe» dans le langage. Le groupe a réussi à ce que l'opinion publique admette cette devise qu'Annie Becquer, «l'auteure» en tête du générique nous a recommandée dans son mot de dédicace: «Il faut rendre à César ce qui est à César et à Cléopâtre ce qui est à Cléopâtre.»

Le phénomène est loin d'être limité à la langue française: les journaux italiens, entre autres, ne cessent de jongler avec «il ministro» et «la ministra» en parlant de leurs femmes distinguées, pour ne citer que cet exemple. Les nouvelles démocraties ouvrent de manière souvent plus généreuse la voie aux appellations féminines, qui sont sans doute plus fréquentes dans ces sociétés et moins tributaires de la pression de la norme (dans les langues slaves par exemple). Il faut dire d'ailleurs que la Suisse, le Québec et la Belgique devancent la France en féminisant plus et depuis plus longtemps: les formes féminines semblent d'ailleurs bien fonctionner dans la pratique langagière de ces pays. Le premier ministre français a tenu à promouvoir la féminisation en un premier lieu dans les cas où le féminin est d'usage fréquent (circulaire du 6 mars 1998) et dernièrement dans la préface à l'ouvrage mentionné ci-dessus. Avec cet élargissement du débat, la féminisation cesse d'être une question idéologique, elle a quitté le domaine symbolique pour se trouver à l'heure actuelle en pleine gestation linguistique.

Le Guide est à la fois une réflexion linguistique qui réussit à mettre en évidence la complexité de la question de façon cohérente et un vaste répertoire de termes masculins / féminins dont la nomenclature dépasse 2000 entrées. L'aspect théorique de la question est exposé essentiellement dans le chapitre qui parle des «difficultés». Si la féminisation pose peu de problèmes sur le plan morphologique, des blocages existent,

1 Cf. Un ouvrage exhaustif à ce sujet est paru récemment: A. Becquer, B. Cerquiglini et al. (1999): Femme, j'écris ton nom...Guide d'aide à la féminisation des noms de métiers, titres, grades et fonctions. 
relatifs à «l'homonymie», «l'euphonie», aux notions de «neutre» et de «générique» et au sentiment de «dévalorisation» liée à certains morphèmes dérivationnels.

Dire que balayeuse représente un homonyme gênant pour deux sémèmes différents (métier de femme et outil mécanique) est un argument affaibli par le fait que le métier existait avant la machine. Il est très curieux que l'ambiguïté correspondante pour les noms masculins ne semble pas poser de problème: le cas du néologisme lecteur (de cassettes) par exemple. Sans parler du «dernier mot» qui échoit au contexte; car c'est ce dernier qui garantit la levée de toute ambiguïté de ce type. Quant aux noms désignant les femmes dans leur statut conjugal et professionnel (ambassadrice), cette homonymie tend à disparaître ${ }^{2}$.

Peu probants sont également les obstacles de dérivation dus au critère euphonie / dysphonie, fréquemment évoqué par les opposants à la féminisation. Un critère plus ou moins arbitraire et subjectif, car la néologie est cacophonique surtout quand elle dérange.

Le Guide souscrit dans une large mesure au sentiment de la péjoration lié à certaines finales (surtout-esse). Or la péjoration est une réalité vague qu'il faut savoir relati$v$ viser $^{3}$. Nos recherches dans le domaine de la suffixation en français non conventionnel nous ont amené à conclure que la dépréciation est plus ou moins limitée à une seule finale: -asse. Et c'est justement la cohabitation avec des suffixes dits «déformateurs» de la langue non normative qui finit par promouvoir en quelque sorte les «bêtes noires» du vocabulaire normalisé, en neutralisant leur dimension dépréciative. La finale -esse par rapport à -arde, -asse, -ouse, -ouille, -oche etc. est un suffixe non marqué. Le français familier et argotique est d'ailleurs traditionnellement favorable à la féminisation: rigolo/rigolote, filou/filoute, loulou/louloute (dès Balzac), beur/beurette etc. Il est regrettable d'ailleurs que le Guide ait renoncé à ces registres qui représentent une composante vivante dans le système linguistique global. Pourquoi proposer une clown si l'on a le choix entre clownesse et clownette. La jockey pourrait suivre le modèle et devenir une jockette. Une gourou sera mieux intégrée au sytème français en imitant l'exemple de voyoute, filoute et louloute. Une gouroute nous semble plus acceptable qu'une gourelle (trop rapprochée du verbe se gourer).

Les auteurs du Guide constatent à juste titre que l'argument grammatical de l'emploi supposé neutre et générique du masculin est dépassé: le français les utilise pour traduire une visée générale. Mais il faut laisser la langue jouer «très finement de l'opposition du générique et du spécifique ${ }^{4} \gg$.

2 Cf. J. Rey-Debove (1998).

Une évolution dans ce sens est tout à fait réelle et fait partie de notre vécu: Madame Françoise Bourolleau qui vient de quitter ses fonctions en Slovénie a réalisé sous nos yeux le passage de l'ambassadeur à l'ambassadrice (cf. ses cartes d'invitation).

3 Cf. V. Pogačnik (1983): «Structuration des suffixes quantificateurs en français non conventionnel», Linguistica, XXIII.

4 Cf. Guide, p. 39

«Dans cet hôpital, les fonctions de chirurgien (générique) sont occupées par une chirurgienne (spécifique)». 
Le cadre théorique ne devrait pas oublier la leçon très utile de Danielle Corbin en matière de la morphologie dérivationnelle. L'intuition des locuteurs et plus particulièrement celle des enfants ${ }^{5} s^{\prime}$ appuie en effet sur la «compétence dérivationnelle» et le «savoir lexical conventionnel» les amenant à former des dérivés «prédictifs» quoiqu'agrammaticaux ${ }^{6}$. Nous ajouterions ici une autre catégorie de locuteurs: les apprenants et les francophones étrangers. Moins compétents ils disposent d'une somme d'informations suffisante pour faire les mêmes «fautes» que les enfants locuteurs natifs. Leurs blocages devant les nouvelles formations sont moindres étant donné que de par leur ignorance ils ont beaucoup moins ou juste assez de scrupules. Leur savoir dans le domaine de la stratification sémantique des homonymes/polysèmes se limite au strict minimum: la médecine pour une doctoresse traitante les gênerait, mais une ingénieuse pour une femme qui fait le métier d'ingénieur peut-être moins. L'exemple avancé nous persuade que le critère de la distribution sémantique sera traité de manière différente dans le domaine de la féminisation. Sont gênants avant tout les homonymes sémantiquement très éloignés (personne/activité), alors que les polysèmes davantage rapprochés, le sont moins (personne/machine). On pourrait faire des enquêtes intéressantes dans ce domaine.

En matière de diachronie, le Guide nous rappelle que les désignations des femmes dans leurs activités très variées étaient féminisées à l'aide de suffixes appropriés. Cet héritage du latin a été négligé par la société du $\mathrm{XIX}^{\mathrm{e}}$ siècle qui sous un masque démocratique montrait par là son vrai visage conservateur: les postes de prestige et d'autorité étant occupés par des hommes, l'effacement de la personne sexuée s'est généralisée, avec la seule exception des noms désignant l'épouse. Aujourd'hui la situation est renversée: l'ambassadrice, la mairesse, la préfète et surtout la pharmacienne s'utilisent de plus en plus pour les fonctions réelles des personnes.

Les règles dérivationnelles présentées dans le Guide suivent pour la plupart le modèle des milieux francophones en dehors de l'Hexagone. Elles semblent à première vue plus que logiques, mais rencontrent chacune des cas difficiles:

1. la féminisation implique l'utilisation d'un déterminant féminin (la députée). Mais: la maître (à la cour)?

2. la forme féminine des masculins se terminant par -e, reste identique (la diplomate). Mais: une maire ou une mairesse, une maître ou une maîtresse, une poète ou une poètesse, une peintre ou une peintresse? Nous opterions sans hésitation pour poètesse et mairesse, car la dimension depréciative ne nous paraît pas pertinente. L'est-elle encore dans Suissesse? Quant à l'épouse du maire, elle peut renoncer à une appellation particulière. La maître et la maîtresse sont également problématiques: peut-être finira-t-on par l'emploi généralisé de maîtresse (comme

5 D. Corbin (1987), pp. 608-611.

6 Ibid., pp. 79-80. 
pour une enseignante), les maitresses du coeur cédant le pas à un nouvel euphémisme - les amies.

3.1. l'adjonction d'un $-e$ à la finale consonantique est gênante dans: commise (à cause de l'homonymie avec faute commise), écrivaine (finale éminemment dérangeante car très rare et trop proche de vaine adj. f. et veine n. f.), substitute. Ne pourraiton pas prévoir une commie, une substitue? L'écriveuse nous semble la seule solution possible pour femme écrivain: il s'agirait d'une forme non connotée par rapport à écrivailleuse et scripteuse.

Le maintien des épicènes n'est peut-être pas nécessaire dans les cas de chef, clerc et conseil. Cheffe et conseille à l'écrit pourraient compenser la défaillance suffixale. Clerque, pratiquée en Belgique nous semble acceptable, matelote répondrait à cheminote, qui n'étonne plus personne, mannequine (malgré l'usage bien ancré du masculin) à arlequine. Avec mannequine au féminin on soulignerait d'ailleurs la masculinisation de manequin. Témoin représente un problème dérivationnel presque insurmontable: cependant témoignante est envisageable, et sans doute préférable à témoigne, témoine? Les féminins de médecin et de marin ne sauraient être en aucun cas médecine et marine à cause d'une distribution sémantique foncièrement différente. On peut avoir recours à une analogie de la série pharmaco-, pharmacienne avec médico-, médicinal, et proposer médicienne à côté de doctoresse (de toubibe dans le français avancé), de traitante et de consultante. Marinière, quoique réservée à d'autres emplois assez restreints, côtoierait bâtelière, jardinière etc.

3.2. Le couple -eur/-euse est bien ancré dans la langue (cf. les dérivés à partir d'une base anglaise: footballeuse, basketteuse, dispatcheuse, supporteuse), si bien que nous ne voyons pas d'utilité d'envisager des féminins en -eure, réservés aux emplois adjectivaux, peu «jolis» dans les dérivés substantivaux, et surtout peu logiques puisqu'il s'agit à l'origine de comparatifs. Il convient d'ajouter que le relatif succès des féminins se terminant par -eure en québécois est sans doute dû à l'influence très proche du milieu anglophone où la distinction entre author-homme et author-femme n'est pas pertinente. Pourquoi entrepreneure, si entrepreneuse figure dans le Petit Robert? Assesseuse est plus acceptable qu'assesseure, malgré la série de sifflantes. Pourquoi gouverneure et non gouverneuse? La forme québecoise annonceure est arbitraire puisqu'il existe un dérivé bien établi - annonceuse. Proviseuse peut s'appuyer sur réviseuse et reviseuse proposées par le Guide, à moins qu'on ne lui préfère provisoresse qui s'inscrirait dans la série défenderesse, demanderesse, venderesse (du vocabulaire juridique), doctoresse (parfaitement conservée) et celle des formations latentes parmi lesquelles rectoresse qui nous semble avoir plus de chances que professoresse. Si seulement la professoressa de l'italien était un peu plus proche des locuteurs français! Il faut avouer que le féminin de professeur reste, à cause de sa fréquence considérable 
dans la pratique langagière quotidienne, le problème $n^{\circ} 1$ de la féminisation: avec lui on bute indéniablement contre une difficulté formelle réelle. La professeure étant à nos yeux plus ou moins un barbarisme, seule peut-être la prof $(f) e$, après une promotion sociale, a des chances de s'imposer définitivement. Car professeuse sonne mal tout comme censeuse à cause d'une redondance gênante des $\mathrm{s}$. La même gêne est décisive dans le blocage absolu devant des formations non réalisées et non réalisables prédécesseuse (on lui préférera devancière) ou possesseuse (peu nécessaire puisqu'elle peut être suppléée par propriétaire, tenante ou détentrice).

3.3. La règle liée au couple -teur/-trice rencontre des difficultés à son tour. La formation de noms féminins en -trice paraît en effet plus probable lorsque la famille lexicale connait un verbe correspondant au nom, ce premier ne comportant pas de - $t$ dans sa base (calculer - calculateur/calculatrice) ou un substantif corrélé au nom se terminant par -tion, -ture ou -torat (édition - éditeurléditrice, lecture lecteur/lectrice, tutorat-tuteur/tutrice). Mais ces contraintes ne sont pas toujours respectées:

- doctrice a cédé le pas à doctoresse (malgré l'existence de doctorat !)

- autrice ne s'appuie sur aucun des éléments cités. Malgré les réticences exprimées par les auteurs du Guide préférant l'auteure à l'aut(h)oresse et à l'autrice, nous pensons que cette dernière a le plus de chances de s'imposer définitivement.

- supportrice est formée sur les masculins supporter (forme anglaise) ou supporteur (forme française) malgré le fait qu'on ne peut pas exclure un verbe latent (et déjà pratiqué en français) supporter comportant un - $t$. La langue a donc des raisons que le raisonnement linguistique méconnaît. Notons ici la distribution heureuse: repporteur/repporteuse en face de rapporteur/rapportrice.

- sculptrice nous semble avoir moins de chances de survie que sculpteuse qualifiée d'obsolète par le Guide. Ici la règle voulant qu'il n'y ait pas de $-t$ dans le verbe s'avère fondée.

4. Reste à éclaircir les solutions possibles de la masculinisation. Comme le constate à juste titre J. Rey-Debove (1998) «c'est la finale du mot, souvenir de son étymologie, qui gêne l'harmonisation du genre et du sexe». Le problème est parfois insurmontable, notamment dans le cas des emprunts: estaffette, sentinelle, vedette. Mais il l'est également pour certains termes très français, liés à la vie militaire où l'attitude dépréciative vis-à-vis des novices est fortement présente et marque certaines lexies: recrue, bleusaille qui sont passées du collectif au singulier par synecdoque, passage encore sensible dans canaille et charogne. Une série de féminins de caractérisation déterminant les individus-hommes est à rapprocher de ce type de lexique: crapule, ordure, frappe, gouape, brute, vache et arsouille (ce dernier change de genre selon l'âge: une jeune arsouille, mais un arsouille mûr). Dans tous ces cas, c'est la dimension sémantique qui bloque la masculinisation autant que l'aspect très féminin de la forme. 
On pourrait avancer que pour les termes plus «techniques» le déterminant finira par changer: un estafet[te] (ou même estafait ?), un recru[e], un sentinel[le].

Dans le sens opposé, c'est-à-dire dans les cas de noms d'aspect masculin et de genre féminin une paradigmatisation à long terme est à prévoir:

«script $\mathrm{m}$. (aujourd'hui féminin) / scripte f.»

«mannequin $\mathrm{m} . /$ mannequine f.»

«star m. / stare f.»

«starlet $\mathrm{m}$. / starlette f. »(basculement déjà opéré sur le plan formel)

La masculinisation ne devrait présenter aucun obstacle d'ordre fonctionnel dans la dérivation régressive du type:

ouvreuse (de cinéma) - ouvreur

jardinière (d'enfant) - jardinier

Le Guide ne prévoit pas chambrier et encore moins cantateur et ballerin qui paraissent peut-être humoristiques, mais qui auraient été très pratiques et finalement peu dérangeants, tout au moins pour les locuteurs «candides» et plus «disponibles».

Pourquoi ne pas accepter sage-homme proposé pour remplacer le savant maïeuticien: mais aide-accoucheur serait plus approprié et surtout plus simple.

En conclusion on constatera que le maintien du masculin a peu de justifications. La féminisation est possible non seulement parce que les outils grammaticaux qui fonctionnaient dans l'ancienne langue sont toujours opérationnels mais aussi parce qu'on découvre de nouveaux moyens à l'échelon synchronique. Il est vrai toutefois que pour certains mots qui malheureusement sont souvent très fréquemment employés, la difficulté formelle de féminisation est réelle (p. ex. témoin, successeur). La difficulté est d'autant plus insurmontable qu'elle se combine souvent avec un blocage qui vient de la distribution sémantique:

- rectrice étant réservée à la plume de la queue d'oiseau, il convient de se contenter de rectoresse pour le titre qui sera proche de doctoresse.

- la médecine et la critique sont des activités, il faut donc chercher une nouvelle dénomination pour les femmes qui les exercent (une médicienne et une criticienne, plus «relevée» qu'une critique?).

- ingénieuse et commise fonctionnent comme adjectif / participe et on ne saurait les substantiver à tout prix. Si une commie (et non une commis proposée dans les autres pays francophones) nous paraît acceptable, il n'y a rien d'approprié pour la femme ingénieur. Des propositions sont cependant possibles: une ingéniaire et dans l'éventualité d'un épanouissement du suffixe -(c)ienne également une ingénicienne (?). 
Les formes en -eure sont contre «l'esprit» de la langue. Elles déséquilibrent l'opposition sémantico-structurelle de deux suffixes foncièrement opposés, qui néanmois cohabitent sur le plan formel, sauf justement pour la marque du genre: blanchisseur $\mathrm{m}$. / blancheur f. D'autre part, elles violent la «chasse réservée» de l'adjectif-comparatif (majeure, mineure, prieure, supérieure). Vouloir les adopter selon le modèle québécois signifierait également céder à la simplification morphosémantique propre à l'anglais. La Suisse et la Belgique préferent d'ailleurs à -eure les solutions avec -trice, -euse, -esse (autrice, auteuse, aut[h]oresse).

La masculinisation présente quelques problèmes, mais en plein essor du mouvement linguistique inverse, elle proposera sans doute des simplifications très acceptables.

\section{Bibliographie}

BECQUER; A. et al. (1999) - Femme, j'écris ton nom... Guide d'aide à la féminisation des noms de métiers, titres, grades et fonctions. Paris: La Documentation française.

CORBIN, D. (1987) - Morphologie dérivationnelle et structuration du lexique (I, II). Tübingen: Max Niemeyer Verlag.

KHAZNADAR, E. (1993) - «Pour une première: La dénomination de la femme dans l'actualité»; Cahiers de lexicologie, 63, pp. 143-169.

Le Nouveau Petit Robert. Paris: Dictionnaires Le Robert, 1993; réimpression et mise à jour, 1994.

POGAČNIK V. (1983) - «Structuration des suffixes quantificateurs en français non conventionnel», Linguistica, XXIII, pp. 125-174.

ReY-Debove, J. (1998) - «Madame "la" ministre», Le Monde, 14 janvier.

WOOLDRIDGE, R. (1999) - «La déféminisation du français»; Prépublication sur Internet à www. chass.utoronto.ca/ wulfric/article/defemin/index.html. 
Povzetek

\section{MOŽNOSTI IN OVIRE ZA FEMINIZACIJO SAMOSTALNIKOV V FRANCOŠČINI}

Prispevek si prizadeva osvetliti pojav feminizacije nekaterih "okorelih" francoskih maskulinskih samostalnikov za poklice, nazive in z njimi povezane funkcije žensk. Družbeno je pojav opravičljiv, čeprav na ideološki in simbolični ravni v francoskih konzervativnih krogih marsikdaj naleti na odpor ob izgovoru, da je "slovnično" neizvedljiv.

Na jezikovni ravni se pokaže, da je ovir za feminizacijo tovrstnih samostalnikov pravzaprav malo, pojavljajo pa se prav pri nekaterih najpogosteje rabljenih besedah, kot so: professeur, médecin, auteur, écrivain, critique, témoin itn. Vendar je tudi tu razsežnost subjektivnega in apriornega razmišljanja močno prisotna. To razmišljanje se največkrat izgovarja na zvočno neubranost (kakofonijo) tvorjenk. Resnično oviro za tvorbo pa pravzaprav predstavlja le poprejšnja "zasedenost" predvidljivih femininskih oblik $z$ bistveno drugačnim pomenom.

Kot dopolnilo k najnovejši francoski monografiji o vprašanju feminizacije članek ponuja nekatere drugačne rešitve, upoštevaje predvsem napovedljive reakcije manj (po)učenih govorcev (otrok in tujcev). Zavzema se tudi za vključitev nenormiranega besedja $v$ to problematiko, saj je leto vitalni del jezikovnega sistema in ponuja nekatere izvirne vzorce, profilira pa tudi razmerja med priponami in besednimi izglasji nasploh, ko gre za tkim. zaznamovanost.

Ugotovitev, da moških samostalnikov na -eur ne kaže feminizirati s pomočjo izglasja -eure se opira na več argumentov: uveljavitev tvorjenk na -eure bi spravila iz ravnovesja opozicionalni odnos med priponama -eur za samostalnike delujočih moških oseb in -eur za samostalnike ženskega spola $\mathrm{s}$ pomenom lastnosti. Tvorba na -eure je $\mathrm{v}$ dosedanjem jezikovnem ustroju omejena na peščico substantiviranih pridevnikov s primerniško vsebino. Prizadevanja za uveljavitev teh oblik so vezana $v$ prvi vrsti na kanadsko francoščino in kažejo na vdor anglosaškega jezikovnega odzivanja.

Zapis na koncu odpira še vprašanje maskulinizacije nekaterih izključno ženskih samostalnikov za poklice, nazive in funkcije. 